\title{
Epistasis of HTR1A and BDNF risk genes alters cortical 5-HT1A receptor binding: PET results link genotype to molecular phenotype in depression
}

\author{
Alexander Kautzky ${ }^{1}$, Gregory M. James, Cecile Philippe ${ }^{2}$, Pia Baldinger-Melich', Christoph Kraus', Georg S. Kranz (1), \\ Thomas Vanicek', Gregor Gryglewski ${ }^{1}$, Annette M. Hartmann ${ }^{3}$, Andreas Hahn', Wolfgang Wadsak $\mathbb{B}^{2,4}$, \\ Markus Mitterhauser ${ }^{2,5}$, Dan Rujescu ${ }^{3}$, Siegfried Kasper ${ }^{1}$ and Rupert Lanzenberger ${ }^{1}$
}

\begin{abstract}
Alterations of the $5-\mathrm{HT}_{1 \mathrm{~A}}$ receptor and BDNF have consistently been associated with affective disorders. Two functional single nucleotide polymorphisms (SNPs), rs6295 of the serotonin 1A receptor gene (HTR1A) and rs6265 of brainderived neurotrophic factor gene (BDNF), may impact transcriptional regulation and expression of the $5-\mathrm{HT}_{1 \mathrm{~A}}$ receptor. Here we investigated interaction effects of rs6295 and rs6265 on 5-HT 1 A receptor binding. Forty-six healthy subjects were scanned with PET using the radioligand [carbonyl- $\left.{ }^{11} \mathrm{C}\right]$ WAY-100635. Genotyping was performed for rs6265 and rs6295. Subjects showing a genotype with at least three risk alleles ( $G$ of rs6295 or A of rs6265) were compared to control genotypes. Cortical surface binding potential ( $\mathrm{BP}_{\mathrm{ND}}$ ) was computed for 32 cortical regions of interest (ROI). Mixed model was applied to study main and interaction effects of ROI and genotype. ANOVA was used for post hoc analyses. Individuals with the risk genotypes exhibited an increase in $5-\mathrm{HT}_{1 \mathrm{~A}}$ receptor binding by an average of $17 \%$ (mean $\mathrm{BP}_{\mathrm{ND}} 3.56 \pm 0.74$ vs. $2.96 \pm 0.88$ ). Mixed model produced an interaction effect of $\mathrm{ROI}$ and genotype on $\mathrm{BP}$ ND and differences could be demonstrated in $10 \mathrm{ROI}$ post hoc. The combination of disadvantageous allelic expression of rs6295 and rs6265 may result in a 5- $\mathrm{HT}_{1 \mathrm{~A}}$ receptor profile comparable to affective disorders as increased 5-HT $1 \mathrm{~A}$ receptor binding is a well published phenotype of depression. Thus, epistasis between BDNF and HTR1A may contribute to the multifactorial risk for affective disorders and our results strongly advocate further research on this genetic signature in affective disorders.
\end{abstract}

\section{Introduction}

The monoamine neurotransmitter serotonin has an essential role in behavior and cognition ${ }^{1}$. Especially for affective disorders serotonin is regarded as the decisive neurotransmitter, implicated in the etiology and course of

\footnotetext{
Correspondence: Rupert Lanzenberger (rupert.lanzenberger@meduniwien.ac.at) ${ }^{1}$ Department of Psychiatry and Psychotherapy, Medical University of Vienna, Wien, Austria

${ }^{2}$ Division of Nuclear Medicine, Department of Biomedical Imaging and Imageguided Therapy, Medical University of Vienna, Wien, Austria
}

Full list of author information is available at the end of the article. the most common neuropsychiatric diseases major depressive disorder (MDD) and anxiety disorders ${ }^{2,3}$.

Consequently, the $5-\mathrm{HT}_{1 \mathrm{~A}}$ receptor has been studied extensively $^{4-7}$. Nevertheless, even fundamental questions as whether reduced or increased $5-\mathrm{HT}_{1 \mathrm{~A}}$ binding should be regarded as neuronal correlates of MDD have not been answered satisfactorily. More recent PET findings have provided some consistency for increased $5-\mathrm{HT}_{1 \mathrm{~A}}$ binding in drug naïve MDD compared to healthy controls, but these results may be dependent on the imaging methodology and specific regions analyzed ${ }^{8-12}$. 
Within the encoding gene HTR1A, the polymorphism rs6265, also known as $C(-1019) \mathrm{G}$, a common variation at the 1019 site upstream of the basal promoter area, has been associated with functional alterations in $5-\mathrm{HT}_{1 \mathrm{~A}}$ receptor signaling ${ }^{13}$. The more common $\mathrm{C}$ allele of this SNP allows binding of the transcriptional factor Deaf1 while the putative risk allele G blocks binding ${ }^{14,15}$. Deaf1 reveals cell specific effects in animal models, such as increasing cortical $5-\mathrm{HT}_{1 \mathrm{~A}}$ receptor binding while decreasing binding in the raphe ${ }^{16}$. Based on this molecular evidence, the $\mathrm{G}$ allele was studied in neuropsychiatric disorders and associated with MDD, bipolar disorder, suicide, as well as neuropharmacological drug response $^{17,18}$. The association of the G allele with MDD was consistently replicated and confirmed in a metaanalysis a few years ago ${ }^{19}$. In addition, the $G$ allele was associated with attenuated response to antidepressant drugs $^{20,21}$.

Brain-derived neurotrophic factor (BDNF), critically involved in brain neuroplasticity, cell survival and axonal growth, has also been shown to influence the serotonergic system $^{22,23}$. The val66met polymorphism, or rs6265, of the $B D N F$ gene modulates BDNF activity by reducing proBDNF in carriers of the less frequent $\mathrm{A}$ or met allele ${ }^{24}$. The A allele has been associated with reduced resilience to stressful life events, aggression, anxiety and memory function. Consequently, it has been studied in a wide range of neuropsychiatric disorders, resulting in mixed findings for affective disorders, schizophrenia and neurodegenerative diseases ${ }^{25}$. Early studies reported the less common A allele to be more frequent in MDD than healthy controls, indicating a possible protective effect for A allele carriers ${ }^{26}$. On the contrary, more recent studies found the A allele to be associated with worse antidepressant treatment outcome, unfavorable clinical characteristics of MDD as psychotic features and suicidality, as well as increased anxiety ${ }^{27,28}$. These latter findings fit in well with the neuroplasticity hypothesis of depression and are backed up by animal models that demonstrated reduced BDNF trafficking in A allele carriers ${ }^{29}$. Ambiguities of the role of BDNF in affective disorders have been discussed for over a decade now. Nevertheless, meta analyses have not been able to disentangle the inconsistencies and reported mostly negative results for val66met and $\mathrm{MDD}^{30,31}$.

Few PET studies have investigated the effect of rs6295 and rs6265 on the serotonergic system (Tables 1 and 2). The G allele of rs6295 of HTR1A was initially associated with increased autoreceptor binding, indicated by elevated binding potential in the dorsal raphe nuclei of depressed patients. However, the same group reported no effects of rs6295 on $5-\mathrm{HT}_{1 \mathrm{~A}}$ receptor binding in a recent update with an expanded sample $9,11,32,33$. Exploiting the reciprocal effect of the rs6295 polymorphism on auto-receptors and heteroreceptors by introducing an alternative measure of non-displaceable binding potential $\left(\mathrm{BP}_{\mathrm{ND}}\right)$ normalized by the dorsal raphe binding, we recently demonstrated effects of rs6295 on $5-\mathrm{HT}_{1 \mathrm{~A}}$ receptor binding in patients with double $\mathrm{G}$ alleles ${ }^{34}$. However, in healthy subjects no effects have been demonstrated so far.

Concerning rs6265 of BDNF, several targets of the serotonergic system were investigated, including serotonin transporter (SERT), $5-\mathrm{HT}_{1 \mathrm{~A}}$, and ${ }_{2 \mathrm{~A}}$ receptor, as well as $5-\mathrm{HT}_{4}$ receptor binding. The less common A allele resulting in methionine was suggested to decrease $5-\mathrm{HT}_{1 \mathrm{~A}}$ receptor binding measured by free plasma concentration binding potential $\left(\mathrm{BP}_{\mathrm{F}}\right)$ while other studies reported negative results based on $\mathrm{BP}_{\mathrm{ND}}{ }^{35-37}$. Recently, the A allele was also associated with increased SERT binding in a large cohort of healthy subjects, while earlier studies reported gender-dependent lower binding in A-carriers or no differences ${ }^{35,36,38}$. No differences were found in $5-\mathrm{HT}_{2 \mathrm{~A}}$ receptor binding ${ }^{39}$. Finally, elevated $5-\mathrm{HT}_{4}$ receptor binding was reported in A allele carriers by the same research group, suggesting a higher brain serotonin activity $^{40}$.

There is evidence for interaction effects of functional genetic variations of BDNF and other genes in the serotonergic system $^{41-43}$. A recent review examining the 5$\mathrm{HT}_{1 \mathrm{~A}}$ receptor in depression suggested interaction between $B D N F$ and HTR1A as an important target for future PET studies ${ }^{32}$. Furthermore, the combination of the risk alleles of rs6265 and rs6295 was associated with treatment resistant depression in a clinical sample ${ }^{44}$. Regarding rs6265 and rs6295, only one PET study has investigated possible interactions of the two SNPs and reported negative findings ${ }^{37}$. Based on these findings we targeted the putative high risk polymorphisms for the 5$\mathrm{HT}_{1 \mathrm{~A}}$ receptor with PET imaging using [carbonyl-11 C] WAY-100635.

\section{Methods \\ Subjects}

All healthy subjects from three previously reported samples collected between 2004 and 2016 for who genotypes for rs6265 and rs6295 were available were pooled for this analysis ${ }^{7,35,41,45,46}$. Due to the lack of a patient sample of adequate size to investigate genetic interaction, only healthy subjects were considered. Consequently, 46 healthy subjects (34 female, 12 male) aged 18-65 (mean age $43.15 \pm 13.08$ ) were available for this cross-sectional neuroimaging study. The lack of single factor effects on 5$\mathrm{HT}_{1 \mathrm{~A}}$ receptor binding was shown before for overlapping cohorts of healthy subjects for rs6295 and rs $6265^{34,35}$. However, surface-based results and particularly the interaction effects of rs6295 and rs6265 have not been previously published. Neuropsychiatric disorders were ruled out for all subjects using the Structured Clinical 

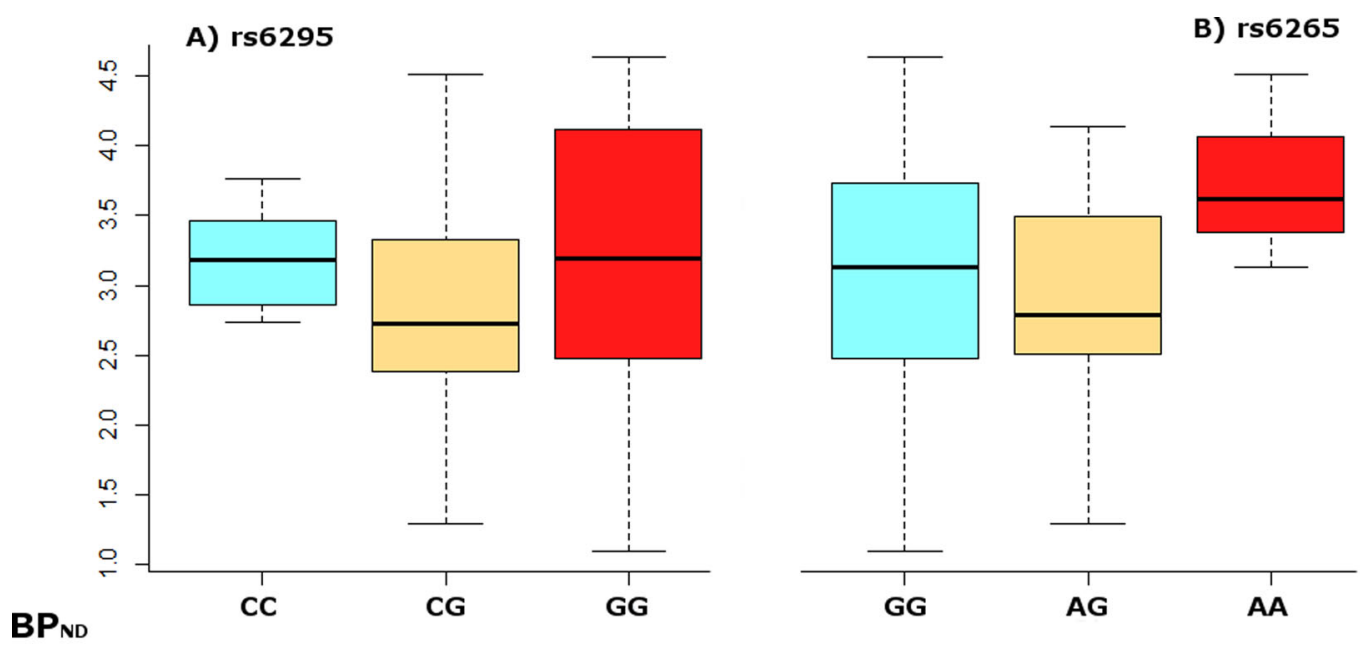

B) rs6265
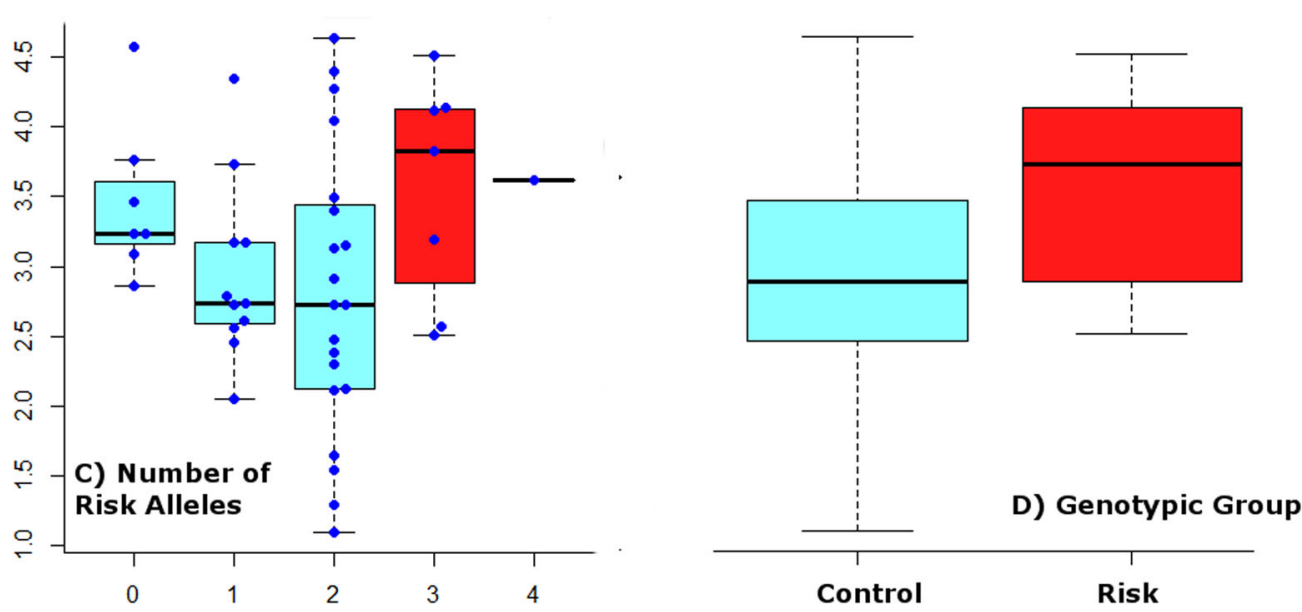

Fig. 1 Boxplot for binding potential ( $\left(\mathrm{BP}_{\mathrm{ND}}\right)$, measured on the $y$-axis. Groups defined by risk alleles are colored red, those by control alleles are turquoise and heterozygote groups are beige. a Mean $B P_{N D}$ is grouped by rs6265 genotype ( $\left.A A=3, A G=17, G G=26\right)$. $b B_{N D}$ is grouped by rs6295 genotype ( $C C=10, C G=19, G G=13)$. c Groups are defined by the absolute number of risk alleles $G$ of rs 6295 and $A$ of rs 6265 , ranging from 0 to 4 $(0, n=7 ; 1, n=11 ; 2, n=20 ; 3, n=7 ; 4, n=1)$. $\mathbf{d} \mathrm{BP}_{\mathrm{ND}}$ is grouped by genotypic group with control $(n=38)$ and risk phenotypes, the latter requiring at least 3 risk alleles $(n=8)$. The difference in mean $\mathrm{BP}_{\mathrm{ND}}$ did not reach statistical significance for any comparison

Interview for DSM-IV type disorders (SCID I + II). All participants underwent a physical and neurological examination including evaluation of clinical history, ECG, routine laboratory analysis, urinary drug, and pregnancy tests. Exposure to any neurotropic drugs or medication over lifetime was an exclusion criterion. All subjects gave written informed consent after receiving detailed oral information concerning the study procedures. The Ethics Committee of the Medical University of Vienna was involved in all studies relevant for this pooled sample and approved all study related procedures. Fo an overview of characteristics of the study sample, please see also Table 1.

\section{Genotyping}

Genotyping procedures were described in previous publications $^{35,46}$. In summary, Ethylene-Diamine-
Tetraacetic-Acid (EDTA) blood samples of $9 \mathrm{ml}$ were extracted from each subject and whole blood was used for DNA isolation with QiaAmp DNA blood maxi kit (Qiagen, Hilden, Germany). The iPLEX assay and the MasSARRAY MALDI-TOF mass spectrometer were used for genotyping, for details please see ${ }^{47}$. Identification of allele specific extension products and definition of genotypes was performed with Typer 3.4 Software (Sequenom, San Diego, CA). Quality requirements for genotyping were defined as an individual call rate above $80 \%$, a SNP call rate over $99 \%$ and over $99 \%$ fit of genotyped CEU trios (Coriell Institute for Medical research, Camden, NJ) with the HapMap database.

\section{Radiochemistry and imaging procedures}

The Division of Nuclear Medicine of the Department of Biomedical and Image-guided Therapy of the Medical 


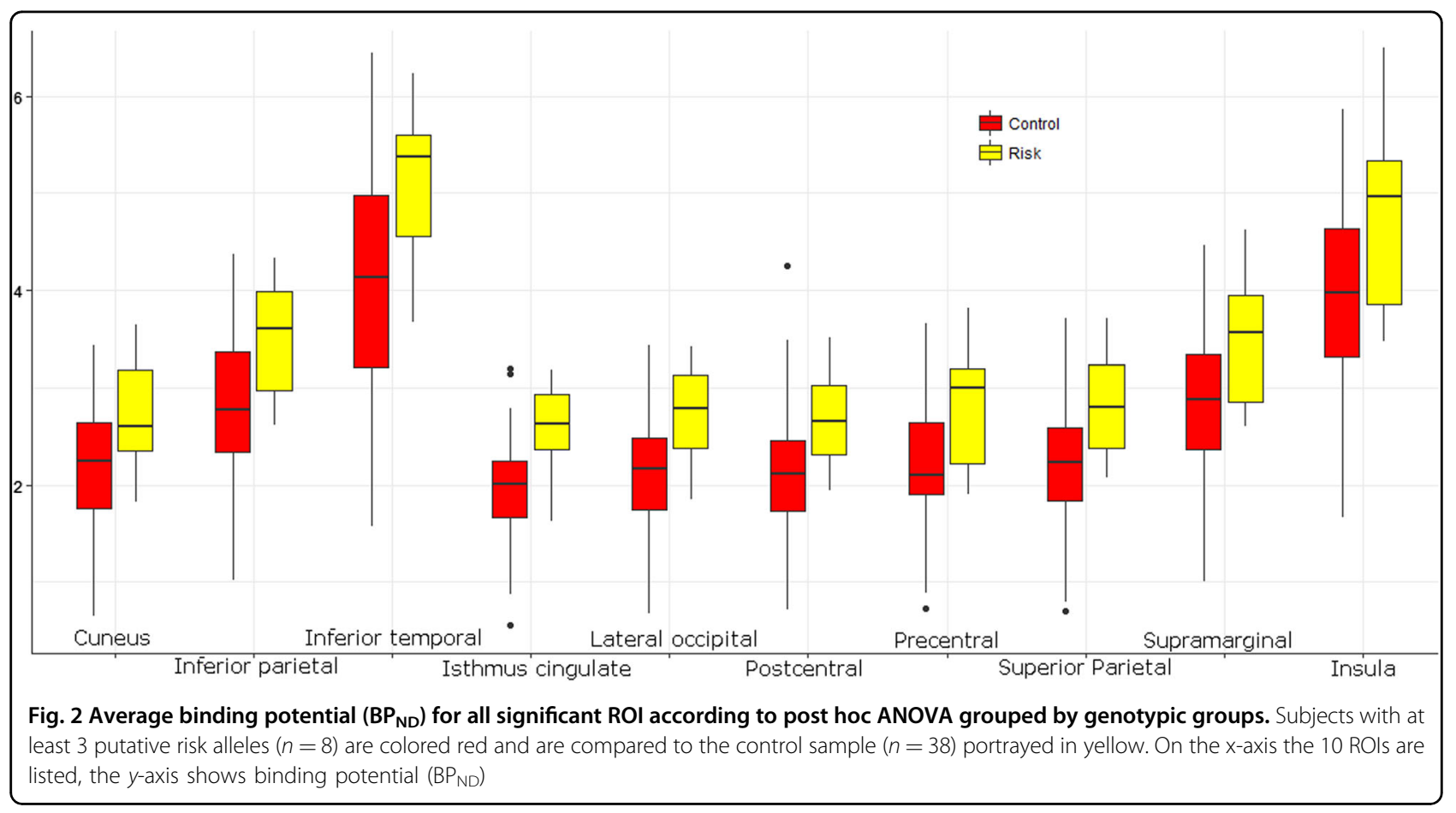

University of Vienna was responsible for all radiosynthetic procedures and provided the PET scanners (General Electric Medical Systems, Milwaukee, WI ${ }^{48}$. The tracer [carbonyl-11C]WAY-100635 was used for all PET scans. Based on the current literature [carbonyl-11C]WAY100635 is the best available radioligand for in vivo $5-\mathrm{HT}_{1 \mathrm{~A}}$ receptor quantification and shows favorable affinity and selectivity ${ }^{49-51}$.

The protocol for measurements required a 5-min transmission scan using a retractable ${ }^{68} \mathrm{Ge}$ rod source to achieve tissue attenuation correction. Next, dynamic emission scan was performed in 3-D mode with mean injected doses of $309.76 \pm 102.46 \mathrm{MBq}$ and molar activity at time of injection of $281.21 \pm 247.52 \mathrm{GBq} / \mu \mathrm{mol}$. The radiochemical purity was above $95 \%$. Data were reconstructed per volume via 35 transaxial sections $(128 \times 128$ matrix) applying a filtered iterative back projection algorithm (FORE-ITER). The spatial resolution was $4.36-\mathrm{mm}$ full-width at half maximum $1 \mathrm{~cm}$ next to the center of the field of view (FOV). Magnetic resonance (MR) images were acquired for 20 of the participants using a 3-Tesla Philips scanner (Achieva) and a T1-weitghted sequence, resulting in 1.56- $\mathrm{mm}$ slice thickness and in plane resolution of $0.78 \times 0.86 \mathrm{~mm}$.

Subjects were placed with their head parallel to the orbitomeatal line guided by a laser beam system to ensure full coverage of the neocortex and the cerebellum in the FOV. A polyurethane cushion and head straps were used to minimize head movement and to guarantee a soft head rest during the whole scanning period.

\section{Data preprocessing}

Freesurfer 6.0 (Harvard Medical School, Boston, USA; http://www.surfer.nmr.mgh.harvard.edu) was applied to reconstruct the cortical surface. T1-weighted MR images served as input whenever available, otherwise the ICBM 152-T1 template was used, after PET images were normalized to standard space, using a tracer-specific template $^{52}$. To detect any mismatches in cortical surface reconstructions, all results were visually inspected. SPM12 (Wellcome Trust Centre for Neuroimaging, London, UK; http://www.fil.ion.ucl.ac.uk/spm) was used for motion correction. This was carried out by the realignment of frame images to median images resulting from a movement-free time period. Subsequently, the coregistration of the median PET images to individual MR images, as well as resulting surface reconstructions was performed. Finally, the motion-corrected dynamic images were combined with registration parameters to partition surface units in vertices.

\section{Kinetic modeling}

Quantification of the cortical $5-\mathrm{HT}_{1 \mathrm{~A}}$ receptor distribution was computed with MATLAB 8.2 (https://www. mathworks.com) using the dynamic PET surface as input. In more detail, the multilinear reference tissue model (MRTM2) was applied to compute the cortical 5- $\mathrm{HT}_{1 \mathrm{~A}}$ receptor availability (Ichise, M. et al. 2003). Thereby, the insular cortex was regarded as high-uptake region while the cerebellar white matter served as a reference region with putatively minimal $5-\mathrm{HT}_{1 \mathrm{~A}}$ receptor concentration ${ }^{51}$. 


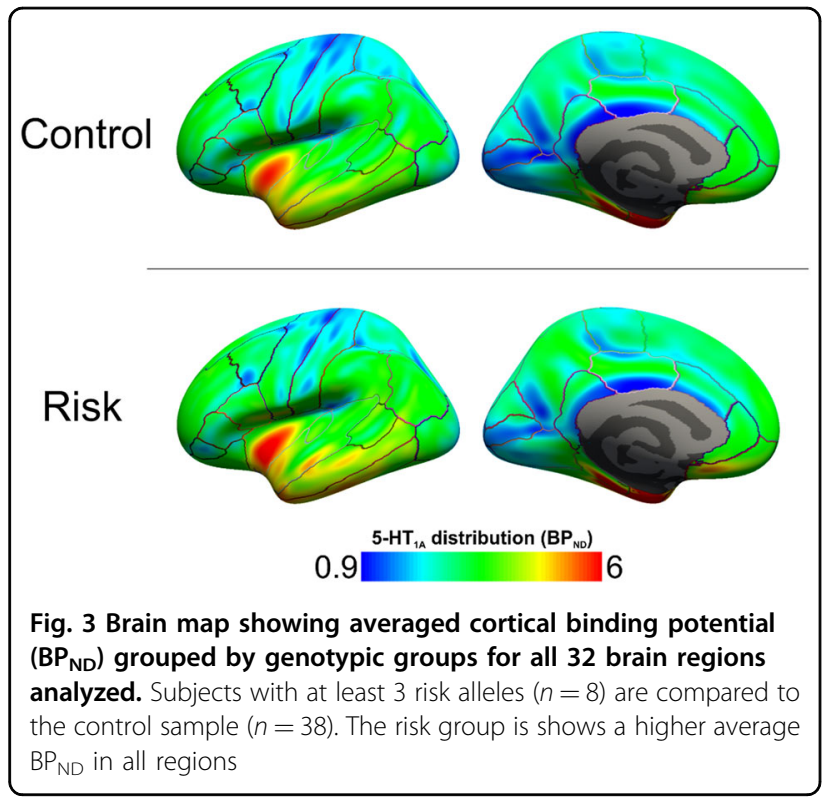

Table 1 Baseline characteristics for the genotypic risk and control groups, respectively

\begin{tabular}{llll}
\hline Baseline characteristics & \multicolumn{2}{c}{ Study sample $(\boldsymbol{n}=\mathbf{4 6})$} & p-value \\
\cline { 2 - 3 } & Risk $(\boldsymbol{n}=\mathbf{8})$ & Control $(\boldsymbol{n}=\mathbf{3 8})$ & \\
\hline rs6295 CC/CG/GG & $0 / 4 / \mathbf{4}$ & $10 / 15 / \mathbf{9}$ & \\
rs6265 GG/AG/AA & $0 / 6 / \mathbf{2}$ & $26 / 11 / \mathbf{1}$ & \\
Sex (male/female) & $3 / 5$ & $9 / 29$ & n.s. \\
Mean age \pm SD & $40 \pm 13.59$ & $43.82 \pm 12.59$ & n.s. \\
Mean BP & $3.56 \pm 0.74$ & $2.96 \pm 0.88$ & n.s. \\
\hline
\end{tabular}

Note: Age and sex did not differ between groups, neither did mean nondisplaceable binding potential

$S D$ standard deviation, $B P_{N D}$ nondisplaceable binding potential

Risk alleles and respective counts in the study sample are indicated by bold values

Subsequently, 32 cortical ROI were delineated based on the Desikan-Killiany atlas ${ }^{53}$.

\section{Statistical analysis}

The statistical software " $R$ " was used for all investigations (cran.r-project.org). Differences between two customized genotypic groups were compared. Specifically, within rs6295 of HTR1A, the G allele has been demonstrated to cause transcriptional dysregulation leading to altered $5-\mathrm{HT}_{1 \mathrm{~A}}$ receptor binding. Thereby, transcriptional effects showed linear increase with the number of $\mathrm{G}$ copies $^{16}$. On the other hand, the A allele of $B D N F$ rs6265 decreases proBDNF levels, disrupting BDNF pathways and putatively also affecting $5-\mathrm{HT}_{1 \mathrm{~A}}$ receptor binding. Therefore, we compared high-risk individuals with at least three risk alleles within the two SNPs, including either
Table 2 Mixed model results, interaction effects are marked with $^{\mathbf{x}}$

\begin{tabular}{|c|c|c|c|c|c|}
\hline $\begin{array}{l}\text { A) Mixed } \\
\text { Model } \\
\text { analysis }\end{array}$ & DF "numerator" & \multicolumn{2}{|c|}{ DF "denominator" } & F-value & $p$-value \\
\hline Group & 1 & 44 & & 3.2249 & n.s. \\
\hline $\mathrm{ROI}$ & 32 & 1408 & & & \\
\hline 247.0292 & $<0.0001$ & & & & \\
\hline $\begin{array}{l}\text { ROI x } \\
\text { genotype }\end{array}$ & 32 & 1408 & & 1.5577 & 0.048 \\
\hline \multicolumn{2}{|c|}{$\begin{array}{l}\text { B) Post Hoc ANOVA } \\
\text { analyses }\end{array}$} & \multicolumn{3}{|c|}{ F-value } & $p$-value \\
\hline \multicolumn{2}{|l|}{ Cuneus } & \multicolumn{3}{|c|}{4.139} & 0.048 \\
\hline \multicolumn{2}{|c|}{ Inferior temporal gyrus } & \multicolumn{3}{|c|}{4.502} & 0.039 \\
\hline \multicolumn{2}{|c|}{ Inferior parietal gyrus } & \multicolumn{3}{|c|}{4.445} & 0.041 \\
\hline \multicolumn{2}{|l|}{ Insula } & \multicolumn{3}{|c|}{4.171} & 0.047 \\
\hline \multicolumn{2}{|c|}{ Isthmus cingulate gyrus } & \multicolumn{3}{|c|}{6.614} & 0.013 \\
\hline \multicolumn{2}{|c|}{ Lateral occipital gyrus } & \multicolumn{3}{|c|}{4.848} & 0.033 \\
\hline \multicolumn{2}{|c|}{ Postcentral gyrus } & \multicolumn{3}{|c|}{4.423} & 0.041 \\
\hline \multicolumn{2}{|c|}{ Precentral gyrus } & \multicolumn{3}{|c|}{4.684} & 0.036 \\
\hline \multicolumn{2}{|c|}{ Supramarginal gyrus } & \multicolumn{3}{|c|}{4.723} & 0.035 \\
\hline \multicolumn{2}{|c|}{ Superior parietal gyrus } & \multicolumn{3}{|c|}{5.441} & 0.024 \\
\hline
\end{tabular}

Note: All mixed model $p$-values are corrected for multiple testing, post hoc analyses are uncorrected. For post hoc results, only significant $\mathrm{ROI}$ are shown $R O I$ region of interest, $D F$ degree of freedom

homozygote subjects for $\mathrm{G}$ allele of rs6295 showing at least one A allele of rs6265 or homozygote subjects for A allele of rs6265 showing at least one $\mathrm{G}$ allele of rs6295. All other genotypic variations were included in the control group. This decision was based on positive findings in combined risk allele carriers in a clinical sample of treatment resistant depression and inconsistent results for imaging studies when either SNP was considered alone $^{34,35,37,44}$. While an increase of effect with the number of unfavorable alleles was reported in preclinical studies, previous PET studies could not substantiate these findings in either patient or healthy cohorts ${ }^{16,32}$. We considered on one hand the small sample sizes available for most PET studies, as well as various compensatory mechanisms extenuating small effects in healthy subjects, and on the other hand the rarity of the most unfavorable genotype with two risk alleles for both SNPs. Thus, comparing subjects with at least three risk alleles to the rest was the preferred approach.

Differences of $\mathrm{BP}_{\mathrm{ND}}$ between genotypic groups were investigated with linear mixed model as included in the "lmne" package of " $R$ " in a ROI based approach ${ }^{54}$. Subject 
served as the random factor and genotypic group, ROI and their interaction served as fixed factors. Sex and age did not affect the model and were hence excluded. In total 32 surface based ROIs were integrated in the model based on the Desikan atlas. For mixed model results a threshold of $p<0.05$ after Bonferroni correction (for number of models, main and interaction effects) was set for significance. For the post hoc analyses for specific ROIs with ANOVA, a $p$-value threshold of 0.05 was determined.

\section{Results}

Risk and control genotype groups did not differ in age or sex. Genotypes for rs6295 and rs6265 were in Hardy Weinberg equilibrium $(p<1)$. The risk group showed overall increased $\mathrm{BP}_{\mathrm{ND}}$ (mean $\mathrm{BP}_{\mathrm{ND}} 3.56 \pm 0.74$ vs. $2.96 \pm$ $0.88 ; F=3.225, p=0.08)$. Mean $\mathrm{BP}_{\mathrm{ND}}$ for genotypes of rs6265 and rs6295, by number of risk alleles and for the risk and control groups is portrayed in Figure 1.

As expected, ROI showed significant effects in the mixed model $(F=247.03, p<0.001$, Bonferroni corrected for the number of models and effects). Furthermore, an interaction effect could be demonstrated for ROI and genotype $(F=1.567, p=0.048$, Bonferroni corrected for the number of models and effects). See also Table 2, section A for mixed model results.

Post hoc analyses with ANOVA were used to identify genotypic effect within specific ROIs and a $p$-value of 0.05 was regarded as significant. Differences between the risk and control genotypes could be demonstrated in 10 regions, as portrayed in Table 3 . These were the cuneus, supramarginal, superior parietal, lateral occipital, isthmus cingulate, inferior temporal, inferior parietal, insula, postcentral and precentral gyrus. See also Table 2, section $\mathrm{B}$, and Figure 2 for post hoc results. For a brain map depicting differences in mean $\mathrm{BP}_{\mathrm{ND}}$ for all cortical $\mathrm{ROI}$ between risk and control groups please refer to Figure 3.

\section{Discussion}

Investigating a large sample of 46 healthy individuals with [carbonyl-11C]WAY-100635, we observed higher 5$\mathrm{HT}_{1 \mathrm{~A}}$ receptor binding by an average of $17 \%$ in the risk group with a genotype of 3 or more putative risk alleles of the two SNPs, rs6265 and rs6295, combined. Our results substantiate the evidence of BDNF and HTR1A polymorphisms val66met and C(-1019)G affecting the serotonergic system in the healthy brain. Increased 5- $\mathrm{HT}_{1 \mathrm{~A}}$ $\mathrm{BP}_{\mathrm{ND}}$ is a well-published phenotype of depressed patients $^{9-11,33}$. Thus, healthy subjects with a risk genotype based on epistasis of rs6265 and rs6295 show similarities with imaging results in MDD.

Both polymorphisms rs6295 and rs6265 show molecular mechanics, which allow them to directly impact the serotonergic system.
For rs6295, the transcription factor Deaf1 is blocked by the $G$ allele at the transcription site, leading to significantly increased $5-\mathrm{HT}_{1 \mathrm{~A}}$ receptor binding in the raphe nuclei but decreased cortical binding in knockout mouse models ${ }^{14,55}$. However, in vivo evaluation of $5-\mathrm{HT}_{1 \mathrm{~A}}$ receptor binding measured by PET in humans could not confirm these mechanisms, as only differences in the dorsal raphe have been reported. A correlation of $\mathrm{BP}_{\mathrm{F}}$ with the number of $\mathrm{G}$ alleles could be observed in 2006 and was initially replicated in $2011^{9,33}$. However, a final evaluation with an expanded sample and refined statistics led the same group to the conclusion that no functional effects can be observed in humans with or without $\mathrm{MDD}^{8}$. Concerning the heteroreceptors, an overall but insignificant increase in $5-\mathrm{HT}_{1 \mathrm{~A}}$ receptor binding in $\mathrm{G}$ allele carriers was observed in the studies described above in cortical areas and in patients with bipolar depression in amygdala and hippocampus ${ }^{32,56}$. These findings were in line with elevated $5-\mathrm{HT}_{1 \mathrm{~A}}$ receptor binding in depressed subjects demonstrated by some studies, however, did not fully converge to the molecular mechanisms reported from animal models regarding $\mathrm{rs} 6295^{9,11,12,33}$. On the other hand, we did not observe significant differences in $\mathrm{BP}_{\mathrm{ND}}$ between rs6295 genotype, unless a transformed outcome parameter $\mathrm{BP}_{\text {Div }}$ was used, normalizing $\mathrm{BP}_{\mathrm{ND}}$ by individual raphe binding potential and therefore exploiting subtle but opposite effects on raphe and projection areas $^{34}$.

There is increasing evidence for direct interactions between serotonin and BDNF ${ }^{57,58}$. Synthesized as a preproneurotrophin, proBDNF is dependent on the removal of the pre-region and requires cleavage by proteases to reach the active form BDNF. The polymorphism rs6265 is especially interesting as the A allele has been demonstrated to reduce proBDNF trafficking and therefore decrease BDNF activity in cell models ${ }^{24}$. Effects seem to be predominant in the central nervous system as cortical neurons and neurosecretory cells, but not endothelial and vascular smooth muscle cells, demonstrated decreased BDNF secretion ${ }^{59}$. These mechanisms could also be observed in animal models, where the homozygous A allele genotype led to anxiety phenotypes ${ }^{29}$. Furthermore, PET studies have been conducted with regards to rs6265 and SERT, 5- $\mathrm{HT}_{1 \mathrm{~A}}, 5-\mathrm{HT}_{2 \mathrm{~A}}$, and $5-\mathrm{HT}_{4}$ receptor binding. Just recently, reduced $5-\mathrm{HT}_{1 \mathrm{~A}}$ receptor binding was reported in A allele carriers while two previous studies reported no effect of rs6265 genotype ${ }^{35-37}$. Furthermore, the largest study so far reported increased SERT binding in A allele carriers, while originally lower binding or absence of genotype effects was reported ${ }^{35,36,38}$. The same group also demonstrated an increase of $5-\mathrm{HT}_{4}$ receptor binding in A allele carriers, suggesting reduced serotonin levels ${ }^{40}$. 
Taken together, these data support functional effects of rs6265 and rs6295 on the serotonergic system, however, the implications and extent of these effects are not yet clear. In synopsis of our studies, we found increased cortical $5-\mathrm{HT}_{1 \mathrm{~A}}$ receptor binding in the risk group, but no effect when either SNP was analyzed separately ${ }^{34,35}$. This interaction effect and the lack of a linear increase of $\mathrm{BP}_{\mathrm{ND}}$ with the number of risk alleles points towards a possible epistasis between HTR1A and BDNF. However, we cannot rule out a simply additive effect of the risk alleles on 5$\mathrm{HT}_{1 \mathrm{~A}}$ binding potential as both SNPs may show significant associations considered separately in sufficiently large sample. In any case, our results indicate healthy controls with combined genetic risk show increased 5$\mathrm{HT}_{1 \mathrm{~A}}$ receptor binding. This is concordant with alterations in depressed patients described by preclinical and imaging findings ${ }^{32,60}$. While the molecular mechanisms of depression are still not sufficiently understood, most of the currently prescribed antidepressant agents target the serotonergic system by blocking the SERT and putatively desensitizing the $5-\mathrm{HT}_{1 \mathrm{~A}}$ autoreceptors while increasing postsynaptic $5-\mathrm{HT}_{1 \mathrm{~A}}$ signaling ${ }^{14}$. In addition, increase of BDNF has been attributed to various antidepressant treatments, including ketamine and $\mathrm{ECT}^{61,62}$. Thus, the two functional polymorphisms rs6265 and rs6295 regulating $5-\mathrm{HT}_{1 \mathrm{~A}}$ signaling and BDNF trafficking may be of relevance for most currently applied antidepressant treatments.

Most importantly, our results differ from the only other PET study on $5-\mathrm{HT}_{1 \mathrm{~A}}$ receptor binding in healthy controls with regards to rs6265 and rs6295, showing decreased binding in A allele carriers but no interaction effect with rs $6295^{37}$. Contrary to previous studies, we focused on cortical ROI due to application of surface based modeling with FreeSurfer. Decisive advantages of this approach have been highlighted recently, most importantly decreasing intersubject variance, a major limiting factor in PET studies investigating the $5-\mathrm{HT}_{1 \mathrm{~A}}$ receptor ${ }^{63,64}$. Surface-based modeling can reduce bias by sustaining cortical geometry, resulting in the gray matter signal being less contaminated with white matter and cerebrospinal fluid ${ }^{65}$.

Besides these differences, divergent findings have partly been explained by methodological variation in PET studies. Different approaches to calculate the concentration of the $5-\mathrm{HT}_{1 \mathrm{~A}}$ receptor have been applied, most notably $\mathrm{BP}_{\mathrm{ND}}$ and $\mathrm{BP}_{\mathrm{F}}$. These refer to the ratio of specifically bound tracer in tissue to either the concentration of free tracer in plasma $\left(\mathrm{BP}_{\mathrm{F}}\right)$ or to the concentration of nondisplaceable tracer in tissue $\left(\mathrm{BP}_{\mathrm{ND}}\right)$, thereby aiming to attain the best estimate of the number of available binding sites, $\mathrm{B}_{\text {avail }}{ }^{66}$. $\mathrm{BP}_{\mathrm{ND}}$ does not require arterial blood sampling and is therefore less invasive. However, $\mathrm{BP}_{\mathrm{F}}$ has been demonstrated to be more favorable as it is independent of a reference region, which could bring bias to the outcome measure. There is insufficient data on direct comparison of $\mathrm{BP}_{\mathrm{ND}}$ and $\mathrm{BP}_{\mathrm{F}}$. One study has shown effects may be different or even opposite, depending on the applied BP variant $^{33}$. Differences were explained by minimal but confounding binding in the reference region, which may also be affected by the genetic polymorphisms investigated. As a control measure, we compared cerebellar white matter time activity curve counts registered during PET measurement between risk and control groups. No significant differences were observed ( $p=0.47, t=0.729)$, indicating that the reference tissue model did not compromise the results due to different binding between genotypic groups in the cerebellum. However, as no direct comparison of the methods was possible, we cannot rule out bias due to these methodological differences.

Another limitation is that our sample has been collected over a decade and is pooled from different PET studies with [carbonyl-11C]WAY-100635. As PET is resource intensive and genetic investigations demand larger samples than usually collected for PET studies due to small effect sizes and stratification by genotypic groups, pooling is often necessary in imaging genetics. Nevertheless, we are confident our results are not significantly biased by pooling procedures. All subjects underwent the same screening procedures concerning somatic and neuropsychiatric disorders and drug naivety, and were measured with the same PET scanner. However, the sample size of 46 subjects is still small and our results must be interpreted as exploratory unless validated in larger, independent samples. It may also be relevant that our sample was skewed towards female sex with roughly $74 \%$ female subjects. While we did not observe significant differences between average $\mathrm{BP}_{\mathrm{ND}}$ of male and female subjects (mean $\mathrm{BP}_{\mathrm{ND}}=3.15$ and 3.01, respectively; $p>$ 0.05 ), effects of as progesterone and testosterone on 5$\mathrm{HT}_{1 \mathrm{~A}}$ binding have been reported previously ${ }^{67,68}$. On the other hand, we did not observe an impact of sex or hormone replacement therapy on $5-\mathrm{HT}_{1 \mathrm{~A}}$ binding in previous PET studies, indicating a less pronounced effect of sex hormones ${ }^{7,69}$.

Apart from these limitations, some other considerations should be discussed.

First, the epigenetic contribution and methylation status have been neglected. The importance of epigenetics, which is still not fully established in neuropsychiatric research, has been consistently demonstrated in the last years. Epigenetic differences explained discrepancies within MDD in monozygotic twins and several methylation markers were suggested as predictors of $\mathrm{MDD}^{70,71}$. There is also evidence that $5-\mathrm{HT}_{1 \mathrm{~A}}$ receptor availability is regulated by a Sp4 site, prone to stress induced hypermethylation, potentially directly impacting $5-\mathrm{HT}_{1 \mathrm{~A}}$ receptor binding PET studies ${ }^{72}$. Consequently, grouping 
solely by genotype without considering methylationinduced inactivation of the target SNPs may be insufficient for some subjects. However, despite strong recommendations to examine methylation in genetic investigations, there have hardly been PET studies accounting for epigenetic effects so far $^{73}$.

Another contributor to divergent findings in imaging genetics could be allosteric heteroreceptor complexes ${ }^{74}$. $5-\mathrm{HT}_{1 \mathrm{~A}}$ receptors have been demonstrated to form heteroreceptor complexes with various G-protein coupled receptors ${ }^{75}$. Tyrosine kinase receptors and tumor necrosis factor receptors relevant to BDNF have also been shown to form heteroreceptor complexes ${ }^{76}$. The lack of findings in direct support of the molecular mechanisms of rs6295 may be explained by FGFR1-5- $\mathrm{HT}_{1 \mathrm{~A}}$ receptor complexes in the raphe nuclei that can disrupt the negative feedback of autoreceptors and eventually lead to $5-\mathrm{HT}_{1 \mathrm{~A}}$ receptor boosting effects in cortical areas ${ }^{77}$. Even though two studies targeting SERT and 5- $\mathrm{HT}_{1 \mathrm{~A}}$ receptor binding with regards to rs6265 reported no interaction effects, implementation of different serotonergic targets in a multivariate model could be mandatory to disentangle the complex genetic scaffoldings of the serotonergic system. However, methodological advances, economizing scanning time and radiation exposure, will probably be necessary to make these models viable ${ }^{78,79}$.

In summary, using PET imaging and [carbonyl-11C] WAY-100635, we provide further evidence the two functional SNPs rs6265 of BDNF and rs6295 of HTR1A impact $5-\mathrm{HT}_{1 \mathrm{~A}}$ receptor binding. Importantly, our results indicate these SNPs do not exert influence on their own, but rather through epistasis, as only subjects with three or more risk alleles showed increased $5-\mathrm{HT}_{1 \mathrm{~A}} \mathrm{BP}_{\mathrm{ND}}$. Thus, we propose that epistasis between HTR1A and BDNF is a control element of the serotonergic system and may be involved in neuropsychiatric disorders as depression. As only healthy subjects were included in this analysis, further investigations in depressed patients are needed to clarify the role of this interaction in MDD. Keeping the limitations in mind, we cannot rule out independence of our findings from methodological issues, such as binding potential computation or the radiotracer used. Epistasis between HTR1A and BDNF may be an important contributor to affective disorders and potentially could become a target for diagnosis and treatment. Our findings are encouraging to further investigate interactions of rs6265 and rs6295 in larger cohorts as implementation of epigenetics and allosteric effects are necessary to fully determine the role of these SNPs in the serotonergic system.

\section{Acknowledgements}

We are grateful to the technical and medical teams of the PET Centre, Medical University of Vienna, especially to K. Kletter, R. Dudczak, L.-K. Mien, J.

Ungersboeck, C. Rami-Mark, and D. Haeusler. Furthermore, we would like to thank A. Höflich, C. Spindelegger, U. Moser, M. Fink, D. Winkler, R. Frey, and P. Stein for medical support, and M. Savli, A. Hahn for technical support. Lastly, we want to thank Ariathney Coyne for her linguistic support. This research was conducted by pooling data from studies supported by grants of the Oesterreichische Nationalbank (Anniversary Fund, project numbers: 11468, 12809) and the Austrian Science Fund (FWF P16549) to R.L. and S.K., respectively, and an intramural grant of the Department of Psychiatry and Psychotherapy (Forschungskostenstelle). Data analyses were funded by the Austrian Science Fund (FWF KLI 551). G.G. is recipient of a DOC-fellowship of the Austrian Academy of Sciences (OeAW) at the Department of Psychiatry and Psychotherapy. The funders had no role in study design, data collection, and analysis, decision to publish, or preparation of the manuscript.

\section{Author details}

'Department of Psychiatry and Psychotherapy, Medical University of Vienna, Wien, Austria. ${ }^{2}$ Division of Nuclear Medicine, Department of Biomedical Imaging and Image-guided Therapy, Medical University of Vienna, Wien, Austria. ${ }^{3}$ University Clinic for Psychiatry, Psychotherapy and Psychosomatic, Martin-Luther-University Halle-Wittenberg, Halle, Germany. ${ }^{4}$ Center for Biomarker Research in Medicine (CBmed), Graz, Austria. ${ }^{5}$ Ludwig Boltzmann Institute Applied Diagnostics, Vienna, Austria

\section{Conflict of interest}

S.K. received grants/research support, consulting fees, and/or honoraria within the last three years from Angelini, AOP Orphan Pharmaceuticals AG, AstraZeneca, Eli Lilly, Janssen, KRKA-Pharma, Lundbeck, Neuraxpharm, Pfizer, Pierre Fabre, Schwabe and Servier. R.L. received travel grants and/or conference speaker honoraria from AstraZeneca, Lundbeck A/S, Dr. Willmar Schwabe GmbH, AOP Orphan Pharmaceuticals AG, Janssen-Cilag Pharma $\mathrm{GmbH}$, and Roche Austria GmbH. Without any relevance to this work, W.W. received speaker honoraria from GE Healthcare, research grants from DSD, BSM Diagnostica and ABX and is a part time employee of CBmed GmbH (Graz, Austria). Without any relevance to this work, M.M. received speaker honoraria from GE Healthcare. P.B.-M. received a travel grant from AOP Orphan Pharmaceuticals AG and speaker honoraria from Janssen. C.K. has received travel grants from Roche Austria GmbH and AOP Orphan. G.S.K. received travel grants from Roche and Pfizer. D.R. reports no potential conflict of interest with relevance to this work. The remaining authors declare no conflict of interest.

\section{Publisher's note}

Springer Nature remains neutral with regard to jurisdictional claims in published maps and institutional affiliations.

Supplementary Information accompanies this paper at (https://doi.org/ 10.1038/s41398-018-0308-2).

Received: 25 July 2018 Accepted: 31 July 2018

Published online: 16 January 2019

\section{References}

1. Lucki, I. The spectrum of behaviors influenced by serotonin. Biol. Psychiatry $\mathbf{4 4}$, 151-162 (1998).

2. Albert, P. R., Benkelfat, C. \& Descarries, L. The neurobiology of depression-revisiting the serotonin hypothesis. I. Cellular and molecular mechanisms. Philos. Trans. R. Soc. Lond. B Biol. Sci. 367, 2378-2381 (2012).

3. Albert, P. R. \& Benkelfat, C. The neurobiology of depression-revisiting the serotonin hypothesis. II. Genetic, epigenetic and clinical studies. Philos. Trans. $R$. Soc. Lond. B Biol. Sci. 368, 20120535 (2013).

4. Fink, M. et al. Lateralization of the serotonin-1A receptor distribution in language areas revealed by PET. Neuroimage 45, 598-605 (2009).

5. Hoflich, A., Baldinger, P., Savli, M., Lanzenberger, R. \& Kasper, S. Imaging treatment effects in depression. Rev. Neurosci. 23, 227-252 (2012).

6. Savli, M. et al. Normative database of the serotonergic system in healthy subjects using multi-tracer PET. Neuroimage 63, 447-459 (2012).

7. Stein, $\mathrm{P}$. et al. The serotonin-1A receptor distribution in healthy men and women measured by PET and [carbonyl-11C]WAY-100635. Eur. J. Nucl. Med Mol. Imaging 35, 2159-2168 (2008). 
8. Kaufman, J. et al. Quantification of the Serotonin 1A Receptor Using PET: Identification of a Potential Biomarker of Major Depression in Males. Neuropsychopharmacology 40, 1692-1699 (2015).

9. Parsey, R. V. et al. Altered serotonin $1 \mathrm{~A}$ binding in major depression: a [carbonyl-C-11]WAY100635 positron emission tomography study. Biol. Psychiatry 59, 106-113 (2006).

10. Parsey, R. V. et al. Higher 5-HT1A receptor binding potential during a major depressive episode predicts poor treatment response: preliminary data from a naturalistic study. Neuropsychopharmacology 31, 1745-1749 (2006).

11. Parsey, R. V. et al. Higher serotonin $1 \mathrm{~A}$ binding in a second major depression cohort: modeling and reference region considerations. Biol. Psychiatry 68, 170-178 (2010).

12. Miller, J. M. et al. Brain serotonin $1 \mathrm{~A}$ receptor binding as a predictor of treatment outcome in major depressive disorder. Biol. Psychiatry 74, 760-767 (2013).

13. Lemonde, S. et al. Impaired repression at a 5-hydroxytryptamine $1 \mathrm{~A}$ receptor gene polymorphism associated with major depression and suicide. J. Neurosci. 23, 8788-8799 (2003).

14. Albert, P. R. Transcriptional regulation of the $5-\mathrm{HT} 1 \mathrm{~A}$ receptor: implications for mental illness. Philos. Trans. R. Soc. Lond. B Biol. Sci. 367, 2402-2415 (2012).

15. Czesak, M., Lemonde, S., Peterson, E. A., Rogaeva, A. \& Albert, P. R. Cell-specific repressor or enhancer activities of Deaf-1 at a serotonin $1 \mathrm{~A}$ receptor gene polymorphism. J. Neurosci. 26, 1864-1871 (2006).

16. Czesak, M. et al. Increased serotonin-1A (5-HT1A) autoreceptor expression and reduced raphe serotonin levels in deformed epidermal autoregulatory factor-1 (Deaf-1) gene knock-out mice. J. Biol. Chem. 287, 6615-6627 (2012).

17. Donaldson, Z. R. et al. The functional serotonin 1a receptor promoter polymorphism, rs6295, is associated with psychiatric illness and differences in transcription. Transl. Psychiatry 6, e746 (2016).

18. Villafuerte, S. M. et al. SSRI response in depression may be influenced by SNPS in HTR1B and HTR1A. Psychiatr. Genet 19, 281-291 (2009).

19. Kishi, T. et al. The serotonin $1 \mathrm{~A}$ receptor gene confer susceptibility to mood disorders: results from an extended meta-analysis of patients with major depression and bipolar disorder. Eur. Arch. Psychiatry Clin. Neurosci. 263 105-118 (2013)

20. Serretti, A. et al. The C(-1019)G polymorphism of the 5-HT1A gene promoter and antidepressant response in mood disorders: preliminary findings. Int $\mathrm{J}$. Neuropsychopharmacol. 7, 453-460 (2004).

21. Lemonde, S., Du, L., Bakish, D., Hrdina, P. \& Albert, P. R. Association of the C $(-1019)$ G 5-HT1A functional promoter polymorphism with antidepressant response. Int J. Neuropsychopharmacol. 7, 501-506 (2004).

22. Martinowich, K. \& Lu, B. Interaction between BDNF and serotonin: role in mood disorders. Neuropsychopharmacology 33, 73-83 (2008).

23. Zagrebelsky, M. \& Korte, M. Form follows function: BDNF and its involvement in sculpting the function and structure of synapses. Neuropharmacology $76 \mathrm{Pt}$ C, 628-638 (2014)

24. Egan, M. F. et al. The BDNF val66met polymorphism affects activity-dependent secretion of BDNF and human memory and hippocampal function. Cell 112, 257-269 (2003).

25. Autry, A. E. \& Monteggia, L. M. Brain-derived neurotrophic factor and neuropsychiatric disorders. Pharmacol. Rev. 64, 238-258 (2012).

26. Ribeiro, L. et al. The brain-derived neurotrophic factor rs6265 (Val66Met) polymorphism and depression in Mexican-Americans. Neuroreport 18, 1291-1293 (2007)

27. Sun, R. F., Zhu, Y. S., Kuang, W. J., Liu, Y. \& Li, S. B. The G-712A polymorphism of brain-derived neurotrophic factor is associated with major depression but not schizophrenia. Neurosci. Lett. 489, 34-37 (2011).

28. Kato, M. \& Serretti, A. Review and meta-analysis of antidepressant pharmacogenetic findings in major depressive disorder. Mol. Psychiatry 15, 473-500 (2010).

29. Chen, Z. Y., Bath, K., McEwen, B., Hempstead, B. \& Lee, F. Impact of genetic variant BDNF (Val66Met) on brain structure and function. Novartis Found. Symp. 289, 180-188 (2008). discussion188-195.

30. Gyekis, J. P. et al. No association of genetic variants in BDNF with major depression: a meta- and gene-based analysis. Am. J. Med Genet B Neuropsychiatr. Genet 162B, 61-70 (2013).

31. Groves, J. O. Is it time to reassess the BDNF hypothesis of depression? Mol. Psychiatry 12, 1079-1088 (2007).

32. Kaufman, J., DeLorenzo, C., Choudhury, S. \& Parsey, R. V. The 5-HT1A receptor in major depressive disorder. Eur. Neuropsychopharmacol. 26, 397-410 (2016).

33. Hesselgrave, N. \& Parsey, R. V. Imaging the serotonin 1A receptor using [11C] WAY100635 in healthy controls and major depression. Philos. Trans. R. Soc Lond. B Biol. Sci. 368, 20120004 (2013).
34. Kautzky, A. et al. The influence of the rs6295 gene polymorphism on serotonin-1A receptor distribution investigated with PET in patients with major depression applying machine learning. Transl. Psychiatry 7, e1150 (2017).

35. Kraus, $C$. et al. Exploring the impact of BDNF Val66Met genotype on serotonin transporter and serotonin-1A receptor binding. PLOS ONE 9, e106810 (2014).

36. Henningsson, S. et al. Genetic variation in brain-derived neurotrophic factor is associated with serotonin transporter but not serotonin-1A receptor availability in men. Biol. Psychiatry 66, 477-485 (2009).

37. Lan, M. J. et al. Genetic variation in brain-derived neurotrophic factor val66met allele is associated with altered serotonin-1A receptor binding in human brain. Neuroimage 94, 33-39 (2014).

38. Fisher, P. M. et al. BDNF val66met association with serotonin transporter binding in healthy humans. Transl. Psychiatry 7, e1029 (2017).

39. Klein, A. B. et al. Cerebral 5-HT2A receptor and serotonin transporter binding in humans are not affected by the val66met BDNF polymorphism status or blood BDNF levels. J. Cereb. Blood Flow. Metab. 30, e1-e7 (2010).

40. Fisher, P. M. et al. BDNF Val66met and 5-HTTLPR polymorphisms predict a human in vivo marker for brain serotonin levels. Hum. Brain Mapp. 36, 313-323 (2015).

41. Baldinger, P. et al. Interaction between 5-HTTLPR and 5-HT1B genotype status enhances cerebral 5-HT1A receptor binding. Neuroimage $\mathbf{1 1 1}$ 505-512 (2015).

42. Pezawas, L. et al. Evidence of biologic epistasis between BDNF and SLC6A4 and implications for depression. Mol. Psychiatry 13, 709-716 (2008).

43. Lin, Z. et al. The interaction of BDNF and NTRK2 gene increases the susceptibility of paranoid schizophrenia. PLOS ONE 8, e74264 (2013).

44. Anttila, S. et al. Interaction between 5-HT1A and BDNF genotypes increases the risk of treatment-resistant depression. J. Neural Transm. 114, 1065-1068 (2007).

45. Baldinger, $P_{\text {., }}$ et al. Effects of Silexan on the serotonin-1A receptor and microstructure of the human brain: a randomized, placebo-controlled, doubleblind, cross-over study with molecular and structural neuroimaging. Int. J. Neuropsychopharmacol. 18, (2015). https://www.ncbi.nlm.nih.gov/pmc/ articles/PMC4360214/.

46. Baldinger, P. et al. Impact of COMT genotype on serotonin-1A receptor binding investigated with PET. Brain Struct. Funct. 219, 2017-2028 (2014).

47. Oeth, P., del Mistro, G., Marnellos, G., Shi, T. \& van den Boom, D. Qualitative and quantitative genotyping using single base primer extension coupled with matrix-assisted laser desorption/ionization time-of-flight mass spectrometry (MassARRAY). Methods Mol. Biol. 578, 307-343 (2009).

48. Rami-Mark, C. et al. Reliable set-up for in-loop (1)(1)C-carboxylations using Grignard reactions for the preparation of [carbonyl-(1)(1)C]WAY-100635 and [(1)(1)C]-(+)-PHNO. Appl. Radiat. Isot. 82, 75-80 (2013).

49. Nics, L. et al. Quantification of the radio-metabolites of the serotonin-1A receptor radioligand [carbonyl-11C]WAY-100635 in human plasma: an HPLCassay which enables measurement of two patients in parallel. Appl. Radiat. Isot. 70, 2730-2736 (2012).

50. Hahn, A. et al. Combining image-derived and venous input functions enables quantification of serotonin-1A receptors with [carbonyl-11C]WAY-100635 independent of arterial sampling. Neuroimage 62, 199-206 (2012).

51. Parsey, R. V. et al. Regional heterogeneity of 5-HT1A receptors in human cerebellum as assessed by positron emission tomography. J. Cereb. Blood Flow. Metab. 25, 785-793 (2005)

52. Fonov, $\mathrm{V}$. et al. Unbiased average age-appropriate atlases for pediatric studies Neuroimage 54, 313-327 (2011).

53. Desikan, R. S. et al. An automated labeling system for subdividing the human cerebral cortex on MRI scans into gyral based regions of interest. Neuroimage 31, 968-980 (2006).

54. Pinheiro, J. C., Bates, D. M. Mixed-effects models in S and S-PLUS. (Springer, New York, 2000).

55. Albert, P. R. \& Fiori, L. M. Transcriptional dys-regulation in anxiety and major depression: 5-HT1A gene promoter architecture as a therapeutic opportunity. Curr. Pharm. Des. 20, 3738-3750 (2014).

56. Sullivan, G. M. et al. Positron emission tomography quantification of serotonin$1 \mathrm{~A}$ receptor binding in medication-free bipolar depression. Biol. Psychiatry 66, 223-230 (2009)

57. Foltran, R. B. \& Diaz, S. L. BDNF isoforms: a round trip ticket between neurogenesis and serotonin? J. Neurochem 138, 204-221 (2016).

58. Jiang, D. G. et al. Serotonin regulates brain-derived neurotrophic factor expression in select brain regions during acute psychological stress. Neural Regen. Res 11, 1471-1479 (2016). 
59. Chen, Z. Y. et al. Variant brain-derived neurotrophic factor (BDNF) (Met66) alters the intracellular trafficking and activity-dependent secretion of wild-type BDNF in neurosecretory cells and cortical neurons. J. Neurosci. 24, 4401-4411 (2004).

60. Richardson-Jones, J. W. et al. 5-HT1A autoreceptor levels determine vulnerability to stress and response to antidepressants. Neuron 65, 40-52 (2010).

61. Bocchio-Chiavetto, L. et al. Electroconvulsive Therapy (ECT) increases serum Brain Derived Neurotrophic Factor (BDNF) in drug resistant depressed patients. Eur. Neuropsychopharmacol. 16, 620-624 (2006),

62. Haile, C. N. et al. Plasma brain derived neurotrophic factor (BDNF) and response to ketamine in treatment-resistant depression. Int J. Neuropsychopharmacol. 17, 331-336 (2014).

63. Matheson, G. J. et al. Reliability of volumetric and surface-based normalisation and smoothing techniques for PET analysis of the cortex: a test-retest analysis using [11C]SCH-23390. Neuroimage 155, 344-353 (2017).

64. Lanzenberger, R. et al. Prediction of SSRI treatment response in major depression based on serotonin transporter interplay between median raphe nucleus and projection areas. Neuroimage 63, 874-881 (2012).

65. Greve, D. N. et al. Cortical surface-based analysis reduces bias and variance in kinetic modeling of brain PET data. Neuroimage 92, 225-236 (2014).

66. Innis, R. B. et al. Consensus nomenclature for in vivo imaging of reversibly binding radioligands. J. Cereb. Blood Flow. Metab. 27, 1533-1539 (2007).

67. Lanzenberger, R. et al. Progesterone level predicts serotonin-1a receptor binding in the male human brain. Neuroendocrinology 94, 84-88 (2011).

68. Stein, P. et al. Relation of progesterone and DHEAS serum levels to 5-HT1A receptor binding potential in pre- and postmenopausal women. Psychoneuroendocrinology 46, 52-63 (2014).

69. Kranz, G. S. et al. Effects of hormone replacement therapy on cerebral serotonin-1A receptor binding in postmenopausal women examined with [carbonyl-(1)(1)C]WAY-100635. Psychoneuroendocrinology 45, 1-10 (2014).
70. Malki, K. et al. Epigenetic differences in monozygotic twins discordant for major depressive disorder. Transl. Psychiatry 6, e839 (2016).

71. Numata, S. et al. Blood diagnostic biomarkers for major depressive disorder using multiplex DNA methylation profiles: discovery and validation. Epigenetics 10, 135-141 (2015).

72. Le Francois, B. et al. Chronic mild stress and antidepressant treatment alter 5HT1A receptor expression by modifying DNA methylation of a conserved Sp4 site. Neurobiol. Dis. 82, 332-341 (2015).

73. Lockwood, L. E., Su, S. \& Youssef, N. A. The role of epigenetics in depression and suicide: a platform for gene-environment interactions. Psychiatry Res $\mathbf{2 2 8}$, 235-242 (2015).

74. Fuxe, K., Borroto-Escuela, D., Fisone, G., Agnati, L. F. \& Tanganelli, S. Understanding the role of heteroreceptor complexes in the central nervous system. Curr. Protein Pept. Sci. 15, 647 (2014).

75. Borroto-Escuela, D. O., et al. Receptor(-)Receptor interactions in multiple 5HT1A heteroreceptor complexes in raphe-hippocampal 5-HT transmission and their relevance for depression and its treatment. Molecules 23, 2018. https:// www.ncbi.nlm.nih.gov/pubmed/29865267.

76. Mehta, H. M., Woo, S. B. \& Neet, K. E. Comparison of nerve growth factor receptor binding models using heterodimeric muteins. J. Neurosci. Res $\mathbf{9 0}$ 2259-2271 (2012).

77. Borroto-Escuela, D. O. et al. Enhancement of the FGFR1 signaling in the FGFR1-5-HT1A heteroreceptor complex in midbrain raphe 5-HT neuron systems. Relevance for neuroplasticity and depression. Biochem Biophys. Res Commun. 463, 180-186 (2015).

78. Sander, C. Y. et al. Neurovascular coupling to D2/D3 dopamine receptor occupancy using simultaneous PET/functional MRI. Proc. Natl Acad. Sci. USA 110, 11169-11174 (2013).

79. Mandeville, J. B. et al. A receptor-based model for dopamine-induced fMRI signal. Neuroimage 75, 46-57 (2013). 